\title{
PHOTOGENERATION AND THERMOGENERATION OF CARBONYL YLIDES: 1,3-DIPOLAR CYCLOADDITIONS OF 2,3-DIARYLOXIRANES TO DIPOLAROPHILES*
}

\author{
G.W. GRIFFIN, J.P.K. WONG, A. A. FAHMI, P.P. UMRIGAR and A.J. MULLER \\ Department of Chemistry, University of New Orleans, New Orleans, LA 70122 (U.S.A.) \\ S. N. EGE \\ Department of Chemistry, University of Michigan, Ann Arbor, MI 48109 (U.S.A.)
}

A series of symmetrically substituted 2,3-diaryloxiranes 1 - 4 (Fig. 1) has been studied as photoprecursors for carbonyl ylides [1 - 3]. The stereochemistry of the adducts obtained on the interception of these $4 n \pi$ transient system with a variety of dipolarophiles provides information on the mode(s) of electrocyclic opening of the oxiranes to carbonyl ylides as well as on the mechanism of the $4 n+2$ cycloaddition processes. While $1 \mathrm{~b}$ undergoes photoinduced ring opening, for example, in the expected disrotatory process, the trans counterpart, as well as $3 \mathbf{a}$ and $\mathbf{3 b}$, behaves anomalously. The stereochemistry of the dipolarophiles in general is preserved in the cycloadducts; this is consistent with a concerted addition process. However, solvent effects, steric hindrance and possibly secondary orbital overlap factors may all play a role in determining the product distribution.

The dipolar species generated photochemically from trans- and cis-2,3bis(2-naphthyl) oxiranes (3a and 3b), unlike those highest occupied molecular orbital ylides derived from the isomeric stilbene oxides, were found to add to electron-rich as well as to electron-deficient alkenes. The cycloaddition reactions of 3 were identified as ground state processes which entail the initial formation of a triplet excited state intermediate. The isomeric bis(2-naphthyl) oxiranes 3 and stilbene oxides 4 also undergo thermally induced cycloadditions. Thermal additions involving such simple unsubstituted oxiranes represent reactions heretofore unreported. In these conversions, only the trans oxiranes behave in a manner consistent with the principles of orbital topology, and we conclude that thermal equilibration of the ylides must precede cycloaddition.

A comparison of the adduct ratios obtained by electron transfer sensitization with dicyanonaphthalene (DCN) using oxiranes 1 and 4 as substrates with the adduct ratios obtained on direct irradiation and thermolysis was conducted [4]. It is evident from such comparisons that regardless of the manner in which C-C bond cleavage is induced a common intermediate, presumably a ground state ylide, is produced before the ensuing interception.

It is noteworthy that oxiranes 5 and 6 bearing electron-deficient substituents such as cyano and carbomethyoxy groups on the three-membered ring undergo direct and energy-transfer-induced photoisomerization. In contrast these substrates proved to be unreactive toward electron transfer photoisomerization using

* Extended abstract of a paper presented at the Xth International Conference on Photochemistry, Iraklion, Crete, Greece, September 6 - 12, 1981. 


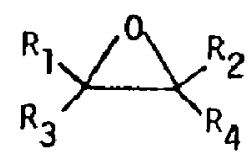

Fig. 1. Structures of the 2,3-diaryloxiranes $1-\mathbf{8}: \mathbf{1 a}, \mathbf{R}_{1} \equiv \mathbf{R}_{4} \equiv \mathrm{CH}_{3}, \mathbf{R}_{\mathbf{2}} \equiv \mathbf{R}_{3} \equiv \mathbf{P h} ; \mathbf{1 b}, \mathbf{R}_{\mathbf{1}} \equiv$ $\mathbf{R}_{2} \equiv \mathrm{CH}_{3}, \mathbf{R}_{3} \equiv \mathbf{R}_{4} \equiv \mathrm{Ph} ; \mathbf{2 a}, \mathbf{R}_{1} \equiv \mathrm{R}_{4} \equiv \mathrm{CH}_{3}, \mathbf{R}_{2} \equiv \mathbf{R}_{3} \equiv 2-\mathrm{Naph} ; \mathbf{2 b}, \mathbf{R}_{1} \equiv \mathbf{R}_{2} \equiv \mathrm{CH}_{3}, \mathbf{R}_{3} \equiv$ $\mathbf{R}_{4} \equiv 2-N a p h ; 3 a, R_{1} \equiv \mathbf{R}_{4} \equiv H, R_{2} \equiv \mathbf{R}_{3} \equiv 2-N a p h ; 3 b, R_{1} \equiv \mathbf{R}_{2} \equiv H, \mathbf{R}_{\mathbf{2}} \equiv \mathbf{R}_{\mathbf{3}} \equiv 2-\mathrm{Naph}$ $\mathbf{4 a}, \mathbf{R}_{\mathbf{1}} \equiv \mathbf{R}_{4} \equiv \mathrm{H}, \mathbf{R}_{\mathbf{2}} \equiv \mathbf{R}_{\mathbf{3}} \equiv \mathrm{Ph} ; \mathbf{4 b}, \mathbf{R}_{\mathbf{1}} \equiv \mathbf{R}_{\mathbf{2}} \equiv \mathrm{H}, \mathbf{R}_{\mathbf{3}} \equiv \mathbf{R}_{\mathbf{4}} \equiv \mathbf{P h} ; \mathbf{5 a}, \mathbf{R}_{\mathbf{1}} \equiv \mathbf{R}_{\mathbf{4}} \equiv \mathrm{CN}, \mathbf{R}_{\mathbf{2}} \equiv \mathbf{R}_{\mathbf{3}} \equiv$ $\mathrm{Ph} ; \mathbf{5 b}, \mathbf{R}_{\mathbf{1}} \equiv \mathbf{R}_{\mathbf{2}} \equiv \mathrm{CN}, \mathbf{R}_{\mathbf{3}} \equiv \mathbf{R}_{4} \equiv \mathrm{Ph} ; \mathbf{6 a}, \mathbf{R}_{\mathbf{1}} \equiv \mathrm{R}_{\mathbf{4}} \equiv \mathrm{CO}_{\mathbf{2}} \mathrm{CH}_{3}, \mathbf{R}_{\mathbf{2}} \equiv \mathbf{R}_{\mathbf{3}} \equiv \mathrm{Ph} ; \mathbf{6 b}, \mathbf{R}_{1} \equiv \mathbf{R}_{\mathbf{2}} \equiv$ $\mathrm{CO}_{2} \mathbf{C H}_{3}, \mathbf{R}_{3} \equiv \mathbf{R}_{4} \equiv \mathrm{Ph} ; \mathbf{7 a}, \mathbf{R}_{1} \equiv \mathbf{R}_{4} \equiv \mathrm{CO}_{2} \mathrm{CH}_{3}, \mathbf{R}_{2} \equiv \mathbf{R}_{3} \equiv p$-Anis; $\mathbf{7 b}, \mathbf{R}_{1} \equiv \mathbf{R}_{2} \equiv \mathrm{CO}_{2} \mathrm{CH}_{3}$, $\mathbf{R}_{\mathbf{1}} \equiv \mathbf{R}_{\mathbf{4}} \equiv p$-Anis; 8a, $\mathbf{R}_{1} \equiv \mathbf{R}_{4} \equiv \mathbf{H}, \mathbf{R}_{\mathbf{2}} \equiv \mathbf{R}_{3} \equiv p$-NCPh; 8b, $\mathbf{R}_{1} \equiv \mathbf{R}_{\mathbf{2}} \equiv \mathrm{H}_{,} \mathbf{R}_{\mathbf{3}} \equiv \mathbf{R}_{4} \equiv p$-NCPh.

DCN as the acceptor. However, aryl substituent perturbation proved effective in activating the isomerization with DCN. For example, the $p$-methoxyaryl-substituted oxiranes $7 \mathrm{a}$ and $\mathbf{7 b}$ (or their methyl counterparts) undergo facile photoinduced isomerization using $\mathrm{DCN}$ as the sensitizer, presumably as a result of a lowering of the oxidation potential of the oxirane donor.

Thesc examples led us to conclude that elcetron-rich energy transfer sensitizers such as tetramethylbenzidine (TMB) might prove effective in promoting the photoisomerization of 5 and 6 via oxirane radical anion intermediates, i.e. that these oxiranes might serve as efficient electron transfer acceptors. That this is indeed the case has been demonstrated by our ability to achieve photointerconversions of $5 a$ and $5 b, 6 a$ and $6 b$, as well as of the stilbene oxides $8 a$ and $8 b$ using TMB as the donor sensitizer.

It is significant that attempts to trap the radical anions implicated in the isomerization of 5, 6 and 8 have proved fruitless to date with both electron-rich and electron-deficient alkenes. For electron-deficient alkenes, the excited state of the donor sensitizer is conceivably quenched by the electron-deficient alkenes employed which are present in high concentration. Laser spectroscopy studies are currently under way to characterize the transients involved in the isomerizations observed for $5 \mathbf{a}-\mathbf{8 a}$.

The results obtained with electron transfer sensitization of vicinal diaryl oxiranes stimulated our interest in the potential cleavage of other $\mathrm{C}-\mathrm{C}$ bonds flanked by aryl substituents in less complex systems such as substituted bibenzyls under similar conditions.

Our preliminary results show that activated bibenzyls bearing electron donor groups such as $p, p^{\prime}$-dimethylbibenzyl and dimethoxybibenzyl (9 and 10 respectively) undergo electron-transfer-induced (with DCN) photocleavage in the presence of oxygen to give high yields of $p$-tolualdehyde and $p$-anisaldehyde. Cross-over experiments conducted with 9 and 10 in the absence of oxygen proved positive, which suggests that benzyl radicals may be formed as primary products of cation radical fragmentation and back donation. The results of our studies using TMB and bibenzyls bearing electron-deficient sensitizers will also be described.

Another focus of recent research in this area has been heterocyclic oxiranes including pyrazolinone spiroxiranes as well as the corresponding diazopyrazoli- 
<smiles>[R]C1=NN(c2ccccc2)C(=O)C12OC2[AlH2]</smiles>

11

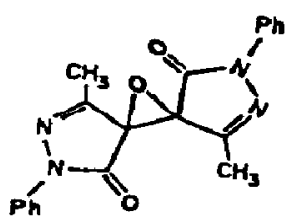

12

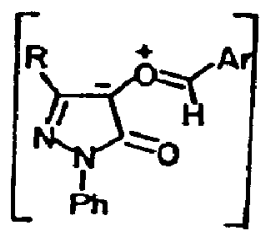

13

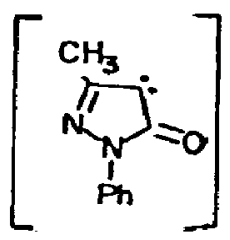

14

Fig. 2. Structures of the heterocyclic spiroxiranes 11 and 12 and the intermediates 13 and 14: a, $\mathrm{R} \equiv \mathrm{CH}_{\mathbf{3}} ; \mathrm{b}, \mathrm{R} \equiv \mathrm{Ph} ; \mathrm{Ar} \equiv 2-\mathrm{Naph}$.

nones which show unusual behavior. Pyrazolinone spiroxiranes such as 11 (Fig. 2) are prepared by basic peroxide oxidation of the corresponding 4-arylidene-2pyrazolin-5-ones. These substrates, as well as the oxirane 12 obtained from $3,3^{\prime}$-dimethyl-1,1' $1^{\prime}$-diphenyl-[ $\Delta^{4,4}$-bi-2-pyrazoline]-5,5' -dione, open on irradiation at $-195^{\circ} \mathrm{C}$ to carbonyl ylides of type 13. Ylides 13a and 13b are trapped with water and/or methanol. 2-Naphthaldehyde or its dimethyl acetal and 4,5-dihydroxy-pyrazoles are isolated as products of this reaction. Laser flash photolysis (at $337.1 \mathrm{~nm}$ ) of $11 \mathrm{a}$ and 12 at room temperature allows observation of the ylide intermediates with lifetimes of 1.6 and $0.52 \mu \mathrm{s}$ respectively (in benzene); $11 \mathrm{a}$ is quenched by methanol with a rate constant equal to $6.0 \times 10^{8}$ $\mathbf{M}^{-1} \mathrm{~s}^{-1}$. Photolysis of 4-diazo-3-methyl-1-phenyl-2-pyrazolin-5-one in methanol gives $Z$ - and $E$-methyl-3-phenylazo-2-butenoates, presumably through the reactive intermediate 14 which may undergo collapse to a ketene before interception with methanol [5]. Recent developments in characterizing the presumed transient(s) in this and other heterocyclic-substituted carbonyl ylide and carbene precursors will be reviewed.

\section{References}

1 N.R. Bertoniere and G.W. Griffin, Formation of carbenes by cycloelimination reactions. In M. Jones, Jr., and R. A. Moss (eds.), Carbenes, Wiley, New York, 1973, Chap. 3, pp. 305 - 349.

2 N. R. Bertoniere and G.W. Griffin, Photochemistry of three-membered heterocycles. In O.L. Chapman (ed.), Organic Photochemistry, Vol. Ill, Dekker, New York, 1973, Chap. 2, pp. $115-195$.

3 G. W. Griffin and A. Padwa, Photochemistry of three- and four-membered heterocyclic rings. In O. Buchardt (ed.), Photochemistry of Heterocyclic Compounds, Wiley, New York, 1976, Chap. 2, pp. $41-122$.

4 A. Albini and D. R. Arnold, Can. J. Chem., 56 (1978) 2985.

5 S. N. Ege, E. J. Gess, A. Thomas, P.P. Umrigar and G.W. Griffin, J. Chem. Soc., Chem. Commun., (1980) 1263. 